\title{
The mab-9 gene controls the fate of $B$, the major male-specific blast cell in the tail region of Caenorhabditis elegans
}

\author{
Andrew D. Chisholm ${ }^{1}$ and Jonathan Hodgkin \\ MRC Laboratory of Molecular Biology, Hills Road, Cambridge, CB2 2QH, England
}

\begin{abstract}
The internal structures of the tail of male Caenorhabditis elegans nematodes are made by the descendants of four cells (B, Y, F, and $U$ ) which divide only in males. These cells are also present in hermaphrodites, where they have minor structural roles in the rectum. Here we show that the gene mab-9 is required for the correct development of two of these male-specific blast cells, B and F. In mutant males, the lineages of B and F resemble those of $Y$ and $U$, respectively. These abnormal lineages lead to grossly defective male tails. We suggest that in mab-9 males the identities of $B$ and $F$ are transformed into $Y$ and $U$, their respective anterior neighbors. The case for the F-to-U transformation is less strong than for the B-to-Y transformation because the wild-type lineages of $F$ and $U$ are very similar. Some mab-9 hermaphrodites are constipated as a result of abnormal rectal structure. This may be the result of an analogous fate transformation. mab-9 worms of both sexes are slightly uncoordinated. We propose that the fates of the four rectal cells are initially specified as two pairs ( $B$ and $Y, F$ and $U$ ) and that the function of mab-9 in both sexes is to differentiate the posterior member of each pair from its anterior neighbor.
\end{abstract}

[Key Words: Caenorhabditis elegans; cell lineage; lineage mutants]

Received May 12, 1989; revised version accepted June 28, 1989.

How are the characteristic fates of cells allocated in development? Mutations that transform cells from one fate into another coherent fate offer a way in to this problem, as they are likely to be affecting genes that are involved in the choice of fate rather than its execution. The homeotic selector genes in Drosophila are the classic example (e.g., Struhl 1982). In the nematode worm Caenorhabditis elegans these questions can be tackled at the single-cell level. Its somatic development has been completely described as an invariant cell lineage (for review, see Sulston 1988). Many mutants have been isolated with developmental (lineage) phenotypes that can be ascribed to transformations of blast cell identity (Horvitz 1988).

One of the most intricate parts of C. elegans development is the formation of the structures of the male copulatory organ or 'male tail' (Sulston et al. 1980). The adult male tail includes a number of sensory organs involved in locating the hermaphrodite vulva, a pair of internal cuticular organs known as spicules (which anchor the male to the hermaphrodite during copulation), and other neurons and muscles used in mating. The internal organs of the male tail are made by cells that only divide in males (the male-specific blast cells $B, Y, U$, and $F$ ); in hermaphrodites they make up the wall of the rectum

'Corresponding author. and do not divide. In the embryonic lineage (Sulston et al. 1983|, F and $U$ are sister cells, whereas B and $Y$ are not related closely; $U$ and $B$ are left-right lineal homologs.

In this paper we describe mutations in a gene, mab-9 (mab stands for male abnormal), which result in primary lineage defects in the posterior two of the male-specific blast cells. mab-9 was first identified by Hodgkin (1983) in a screen for mating-defective males. mab-9 males cannot mate, having grossly deformed tails and lacking most internal tail structures. A preliminary analysis of the tail lineages indicated that the focus of the defect was in the B lineage, and possibly others (Hodgkin 1983). It was suggested that the mutant phenotype could be the result of a partial sexual transformation of the dividing male $\mathrm{B}$ cell towards the nondividing hermaphrodite $\mathrm{B}$.

Here we present evidence that the phenotype of mab-9 is explained more readily as a spatial transformation of the identity of the B cell toward that of $Y$, and probably also a transformation of $F$ toward $U$. This transformation appears to occur in both sexes, the phenotype being more obvious in males because of the greater role of the B blast cell in male development. mab-9 worms of both sexes are also slightly uncoordinated. Therefore, the mab-9 gene functions in both sexes in the specification of the identity of one or two cells in the tail region of the worm, and in the locomotory system (neurons or muscle). 


\section{Results}

\section{Background}

The development of the male tail is entirely postembryonic: at hatching, the tails of male and hermaphrodite worms appear virtually identical. Most of the adult tail derives from postembryonic blast cells that divide in different patterns in the two sexes. Muscles are generated by the mesoblast $M$; the eighteen sensory rays are made by the lateral hypodermal blast cells (V5, V6, and $\mathrm{T}$, bilaterally paired), and the hook and hook sensillum are made by the ventral hypodermal blast cells P9, P10, and P11. Several genes are known which affect the lineages of the hypodermal blast cells, including mab-5 (Kenyon 1986) and mab-3 (Shen and Hodgkin 1988). The internal structures (the spicules and associated neurons) are produced by the blast cells $B, Y, U$, and $F$, which divide only in males and are structural cells in the rectum of hermaphrodites. The arrangement of the internal blast cells in wild-type early $\mathrm{L} 2$ males is shown in Figure 1, $a$ and $b$.

$B$ is the major blast cell, producing 42 cells that make up the spicules, proctodeum, and associated neurons. Y produces 11 neurons: the two postcloacal sensilla 15 cells each) and a preanal neuron (PDA = Y.a). The Y lineage and the early B lineage are shown in Figure 2, a and $d$. The lineages of the two other blast cells $U$ and $F$ are very different from those of $B$ and $Y$. The $U$ and $F$ lineages are similar except for an extra division round in $\mathrm{F}$ (shown in Fig. 4a and c); the neuron types made by $U$ and F appear to be the same (Sulston et al. 1980).

\section{Isolation of mab-9 alleles}

A preliminary examination of the lineages of the malespecific blast cells of mab-9 males, using mutants for the reference allele $e 1245$, had shown that the B lineage was abnormal (Hodgkin 1983). However, it was not known whether $e 1245$ represented the complete loss-offunction phenotype for the locus. To clarify the null phenotype of mab-9, two more alleles were isolated (see Materials and methods). All three alleles are fully recessive. The lineage phenotypes of $e 1245$ and e2410 appear similar when the alleles are placed over a deficency known to span the locus. The penetrance of some other phenotypes in strains containing the three alleles is shown in Table 1. By the criteria of penetrance, e2410 is the strongest allele; $e 1245$, which is a nonsense mutation (see Materials and methods), is almost as strong; $e 2376$ is hypomorphic.

\section{Transformation of $B$ to $Y$ in males}

By several criteria the B lineage in mab-9 males is transformed, to a variable degree, toward a lineage like that of the $Y$ cell, although a perfect $Y$ lineage is only seen occasionally. $\mathrm{Y}$ is always wild type in mab-9. Characteristics of the abnormal $\mathrm{B}$ lineages are detailed below: $\mathrm{B}$
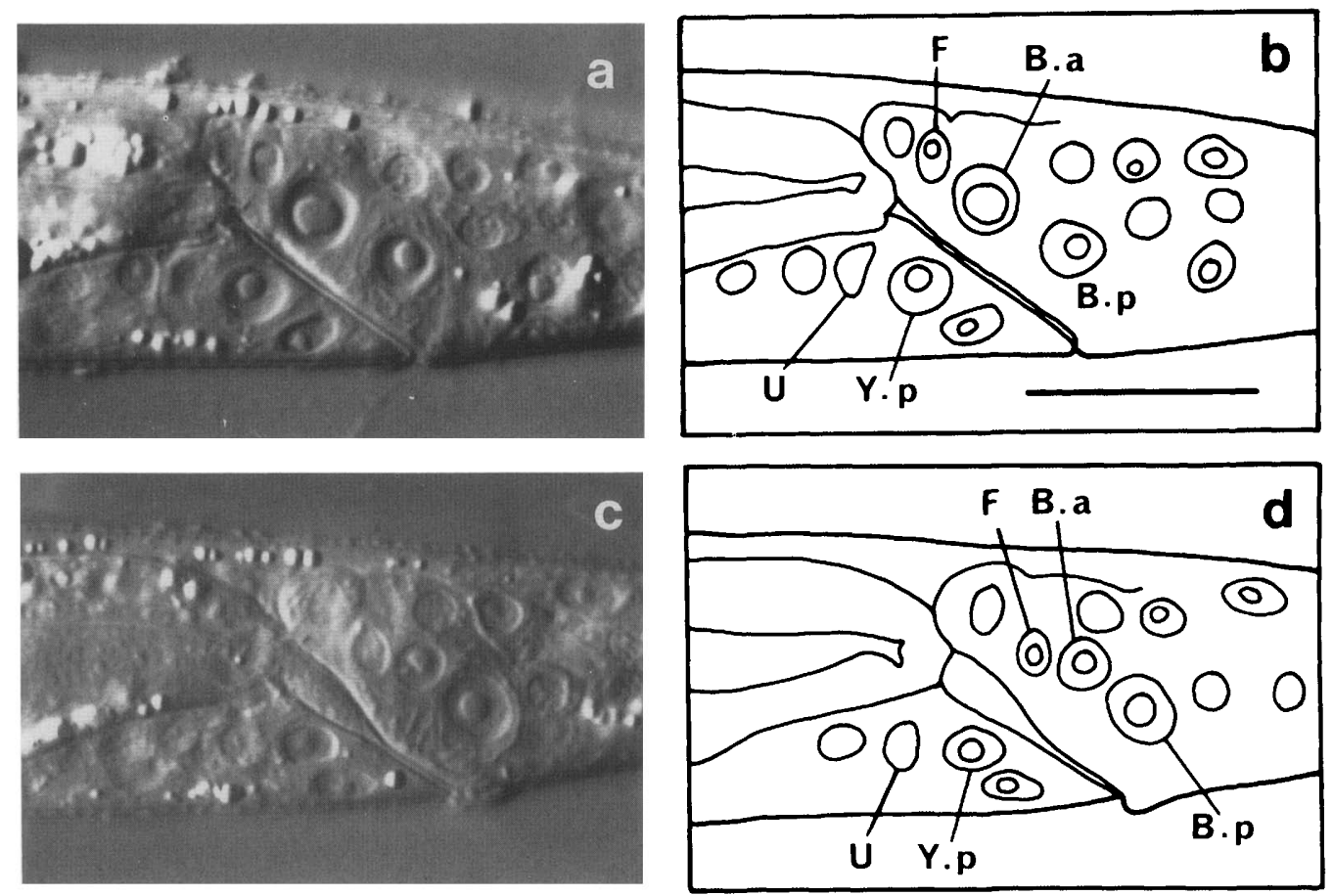

Figure 1. Nomarski photomicrographs and tracings of tails of wild-type and mab-9 mutant L2 males. Anterior is to the left and dorsal is up. Scale bar, $10 \mu \mathrm{m}$. Male animals derive from strains containing him-5(e1490). $(a)$ Lateral view (focal plane in midline) of tail region of wild-type early L2 male. B and Y have divided; note the relative size of the B daughter nuclei /Y.a is just out of the plane of focus). (c) Lateral view, as in $a$, of tail region of a mab-9(e2410) early L2 male. B.a is slightly smaller than B.p. $(b$ and $d)$ Tracings of $a$ and $c$, respectively; the $U$ and $F$ cells are also labeled. 

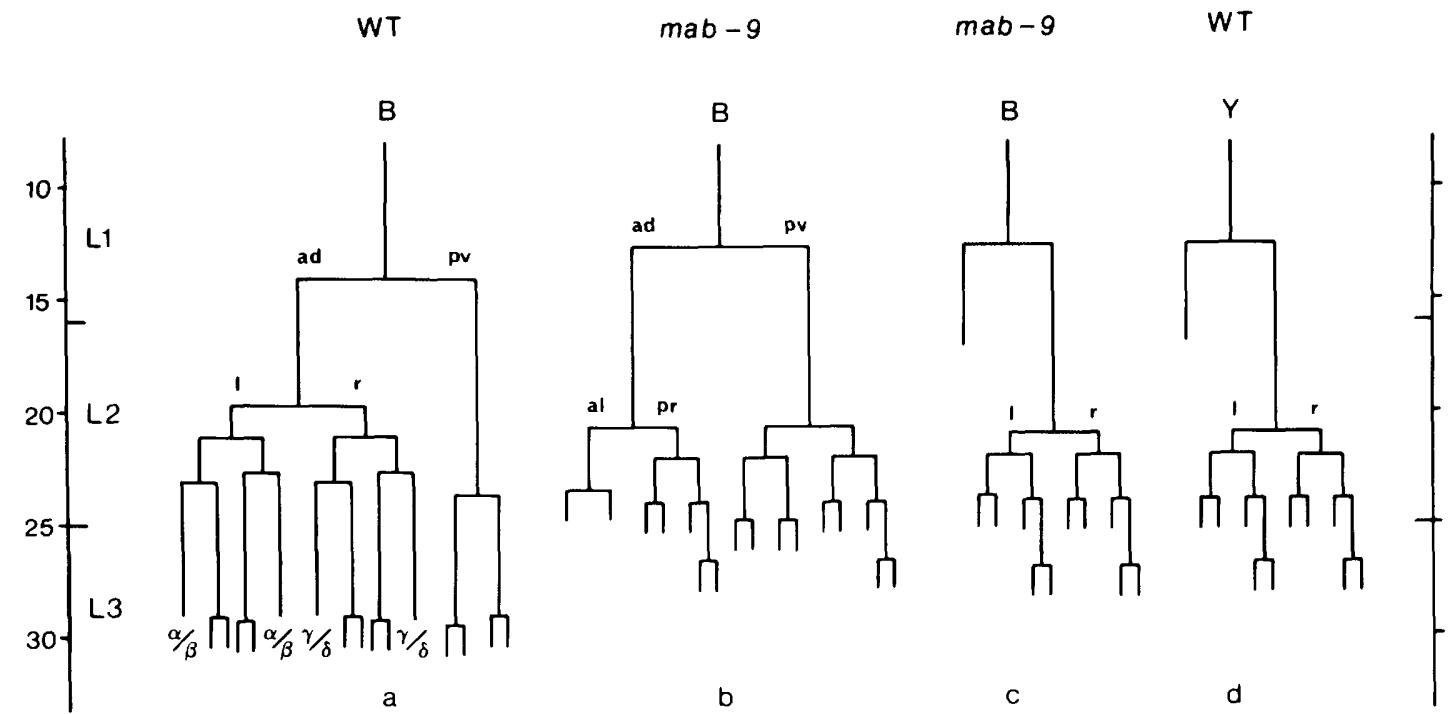

Figure 2. Wild-type and mutant B lineages. Vertical axis is time in hours after hatching, at $20^{\circ} \mathrm{C}$, divided into the four larval stages $\mathrm{Ll}-\mathrm{L} 4$. Branchings represent cell divisions in the anteroposterior axis unless labeled otherwise (anterior is to the left). Division axes are described relative to the main axes of the worm: anteroposterior $(\mathrm{a} / \mathrm{p}\rangle$, dorsoventral $\langle\mathrm{d} / \mathrm{v}\rangle$, left-right $(1 / \mathrm{r})$, or any combination of the above. Cells are named by their lineage ancestry from a founder cell by adding a series of letters representing the sequence of cell divisions giving rise to that cell. Ends of lines represent terminal differentiation. All wild-type lineages shown in this and subsequent figures are taken from Sulston et al. 1980. (a) Wild-type early B lineage. All eight progeny of B.a commence two or three further rounds of division after $\sim 29 \mathrm{hr}$, but the fates of the pairs B.alaa/B.araa (fates $\alpha / \beta)$ and B.alpp/B.arpp (fates $\gamma / \delta$ ) are determined by cell-cell interaction. (b) Typical 'intermediate' B lineage from a mab-9(e1245) male. The following genotypes were lineaged from late $\mathrm{Ll}$ to the L3 molt (numbers for each are in brackets): e1245 (5); e1245/nDf3 $(4) ; e 2376(5) ; e 2410(5) ; e 2410 / n D f 3$ (4). Several partial lineages (up to the L2 molt or early L3) also were done for each genotype. Variability of the mutant B lineage is seen in both strong alleles and the deficiency heterozygotes. The lineages from e2376 were the most weakly transformed: In some animals B.a was still larger than B.p. The other two alleles usually gave patterns like the one shown la partial transformation: $3 / 5 e 1245,2 / 4 e 1245 / n D f 3,4 / 5 e 2410,4 / 4$ $e 2410 / n D f 3$, the lineages becoming more variable after the L2 molt. In l/5 e1245 worms, both B daughters had a neuronal nuclear morphology and did not divide, perhaps indicating a symmetrization to give two Y.a-like fates. In $1 / 5$ e2410 worms a curious hybrid lineage was seen, in which B.a did not divide (and was dorsal to B.p), whereas B.p divided ad/pv (simultaneously with Y.p); B.pa then underwent a lineage resembling that of wild-type B.a, whereas the lineage of B.pp resembled that of Y.p. Proliferation generally did not continue after mid-L3, except in one or two cases in which the B progeny in L3 were large and divided variably in late L3 and early L4 (the time of the late rounds of division in the wild-type B lineage). (c) Strongly transformed B lineage, seen in $2 / 4$ e1245/nDf3 and $1 / 5$ e1245 worms. Apart from minor differences in the axes of division the lineages were identical to those of Y. (d) Wild-type Y lineage.

Table 1. Penetrance of mab-9 phenotypes

\begin{tabular}{|c|c|c|c|c|}
\hline \multirow[b]{2}{*}{ Allele } & \multicolumn{2}{|c|}{ Male phenotypes } & \multicolumn{2}{|c|}{$\begin{array}{l}\text { Hermaphrodite } \\
\text { phenotypes }\end{array}$} \\
\hline & $\begin{array}{l}\text { tail }^{\mathbf{a}} \\
(\%)\end{array}$ & $\begin{array}{l}\text { spicules }^{b} \\
|\%|\end{array}$ & $\begin{array}{l}\mathrm{B} \text { cellc } \\
|\%|\end{array}$ & $\begin{array}{l}\mathrm{Egl} / \mathrm{ill}^{\mathrm{d}} \\
|\%|\end{array}$ \\
\hline$e 2410$ & $58\langle 100\}$ & $98(100)$ & $64(50)$ & $7.0(1564)$ \\
\hline e1245am & $37(114)$ & $98(114)$ & $32(50)$ & $1.7(1805)$ \\
\hline$e 2376$ & $23(101)$ & $82(101)$ & $34 \mid 50)$ & $0.3(1394)$ \\
\hline
\end{tabular}

For each column the percentage mutant is followed by the number of animals scored (in parentheses).

ail morphology: The number of adult mab-9 males with the distinctive "posterior bulge" morphology (Fig. 5b and d) was scored.

b Percentage adult mab-9 males without spicules.

c Nomarski morphology of the B nucleus in L4 or young adult mab-9 hermaphrodites. The percentage with abnormal or neuronal morphology (Fig. 3 b) is shown. An abnormal B correlated well with constipation: 25 of 50 e2410 L4 hermaphrodites and 9 of 50 e1245 were to some degree constipated, with 22 of 25 and 6 of 9 also having abnormal B morphology (and therefore 25 of 50 and 41 of 50 not constipated, with 14 of 25 and 10 of 41 having an abnormal $\mathrm{B}$ ).

d Penetrance of the severely constipated, Egl and ill phenotype in mab-9 adult hermaphrodites. lineages are described as strongly or weakly transformed according to how Y-like they appear. The phenotypes described were seen in mutants for the strong alleles $e 2410$ and $e 1245$ (see Fig. 2).

Timing of divisions In mab-9 males, the early rounds of division in the B lineage are advanced or retarded to become synchronous with the comparable Y-lineage divisions (to within $\sim 20 \mathrm{~min}$ ). This synchronization of division rounds is striking during $\mathrm{L} 1$ and $\mathrm{L} 2$, but becomes less precise later. Wild-type $B$ and $Y$ lineages are shown in Figure 2,a and $d$; typical mutant $B$ lineages are shown in Figure 2,b and c.

Size of cells Normally in mid-L1 males the B cell has a large nucleus and prominent nucleolus. In mab-9 males, $B$ usually has a size intermediate between that of wildtype $B$ and $\mathrm{Y}$. This is the first visible abnormality in mab-9 male larvae. The cells generated by the B lineage by mid-L3 are usually much smaller than normal, and often have a neuronal nuclear morphology, mirroring the neuronal progeny of the Y cell.

Unequal divisions The first divisions of both $\mathrm{B}$ and $\mathrm{Y}$ are unequal (Y.p and B.a are larger than their sisters) and 
asymmetric with regard to the fates of the daughters ('nonequational', in the terminology of Sulston et al. 1983). In mab-9 males, the inequality and the fate asymmetry of the B division are either lost or reversed (see Fig. $1 \mathrm{~b}$ and $\mathrm{c}$. In the former case (weak transformation, Fig. 2b), B.a and B.p are of equal size and undergo similar division patterns; in the latter, B.a is now the smaller and does not divide (like Y.a), whereas B.p executes a Y.p-like lineage (strong transformation, Fig. $2 \mathrm{c}$ ).

Division axes The initial division of $\mathrm{B}$ is oriented anteriodorsally-posterioventrally $(\mathrm{ad} / \mathrm{pv})$. This is usually preserved in mab-9, but in some cases the axis may be oriented $\mathrm{d} / \mathrm{v}$ or even $\mathrm{av} / \mathrm{pd}$; in the latter, the dorsal daughter is always smaller and does not divide. In the wild type, B.a and Y.p divide symmetrically across the midline in early L2 (B.p divides later, along the midline); in mab-9, both B daughters or just B.p divide across the midline, albeit in a skewed fashion. Subsequent division axes become more variable, although in strongly transformed lineages they resemble those of Y.p closely.

Proliferation Normally, Y makes 11 cells and B makes 47 (5 of these die). In strongly transformed B lineages in mab-9, B may make a similar number of cells to $\mathrm{Y}_{\text {; }}$ in worms where B progeny continue to divide in late L3, as many as 20-25 small cells may be generated in the B region, although never as many as in wild type.

Differentiated fates Most of the terminal fates of the wild-type B lineage and all of those of $\mathrm{Y}$ are neuronal, and thus indistinguishable by Nomarski microscopy. No specific antibody markers are available yet for the B lineage neuronal types. However, several B cells are structural (making the spicules and gubernaculum by secreting a sclerotized cuticlel. In mab-9 male tails, spicules are almost never made (see Table 1 ; Fig. 5 b and d). The five B lineage programmed cell deaths in early L4 are unquestionably terminal fates and can be scored unambiguously: they were absent in several mab-9(e2410) and mab-9(e1245) L4 males examined.

To summarize, the particular characteristics of the mutant $B$ lineages suggest that they are the result of a variable transformation of B towards $Y$. The B lineage transformation is sufficient to account for the lack of internal structures in mab-9 male tails.

\section{Abnormal $B$ cells in mab-9 hermaphrodites}

Most mab-9 hermaphrodites are wild type in morphology. However, about $7 \%$ of mab-9(e2410) adult hermaphrodites are severely constipated and are egg-laying defective (Egl). The constipation is the result of blockage at the rectum. The worms appear sickly and have a refractile blob hanging from the cloaca, the result of a partial eversion of the rectum. These phenotypes can be traced to abnormalities in the $\mathrm{B}$ cell of mab-9 hermaphrodites.

In wild-type hermaphrodites, the B cell comprises part of the dorsal posterior wall of the rectum and does not divide. Its nucleus is larger than those of neighboring neurons, and the nucleolus is prominent /see Fig. 3a and
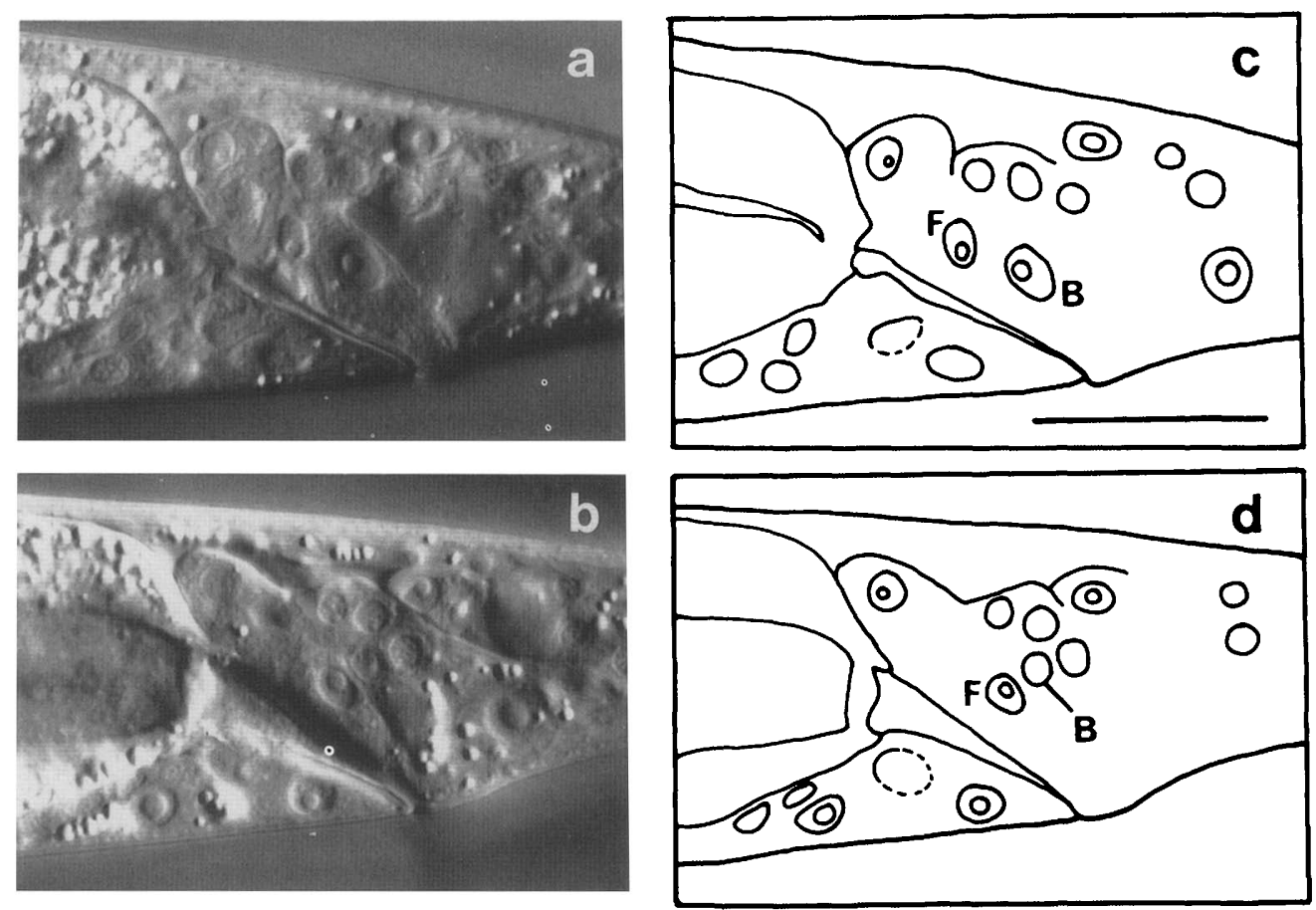

Figure 3. Nomarski photomicrographs and line drawings of $\mathrm{B}$ in wild-type and $m a b-9(e 2410)$ hermaphrodites. Scale bar, $10 \mu \mathrm{m}$. $(a)$ Lateral view, focal plane in midline. Wild-type $L 4$ hermaphrodite. B is larger than $F$ and has a distinct nucleolus. $(b)$ Lateral view, focal plane in midline. mab-9(e2410) L4 hermaphrodite. Notice that B is distinctly smaller and has neuronal nuclear morphology (compare the three neighboring midline neurons with small grainy nuclei). $Y$ has migrated into the preanal ganglion and become the neuron PDA. ( $c$ and $d)$ Tracings of $a$ and $b$, respectively. 
c). In contrast, $\mathrm{Y}$ is a structural cell in $\mathrm{L} 1$, but by $\mathrm{L} 4$ it has moved anteriorly into the preanal ganglion to become the neuron PDA.

The appearance of $\mathrm{B}$ in $m a b-9$ hermaphrodites parallels that of Y. In 36 of 50 mab-9(e2410) L4 hermaphrodites examined, the $\mathrm{B}$ nucleus appeared abnormal: in several of these animals the B nucleus resembled that of a neuron (i.e., small, grainy nucleoplasm and indistinct nucleolus) rather than that of a structural cell; in the others, B was small, shriveled and had no distinct nucleolus (see Fig. $3 \mathrm{~b}$ and d). (The penetrance of these phenotypes for the other two alleles is given in Table 1.) The abnormal appearance of $B$ correlates well with the constipated phenotype: out of the 50 e2410 worms, 25 were constipated, and of those, 22 also had an abnormal B nucleus (the other 14 with abnormal B were not constipated). However, when $B$ was examined in L1 mab-9(e2410) hermaphrodites, its nucleus appeared normal. The $\mathrm{Y}$ cell always appeared normal in mab-9 hermaphrodites.

Can the abnormalities in B account for the constipation, sickness, and Egl phenotypes? To address this, we killed the B cell by laser irradiation /see Materials and methods) in 16 wild-type $\mathrm{L} 1$ hermaphrodite larvae: several of these worms became mildly constipated by adulthood, and one became severely constipated, Egl and very sick. Of $10 \mathrm{Lls}$ in which both $\mathrm{B}$ and $\mathrm{F}$ were killed, one worm became similarly afflicted. These experiments therefore phenocopy the mab-9 hermaphrodite phenotypes, suggesting that they can be ascribed to abnormalities in B. A severely constipated animal stops eating (perhaps sensing the distension of its gut), becomes starved, and consequently begins to retain its eggs (Egl). The penetrance of the phenotypes is low in both the mutants and the kills, showing that B function is almost dispensable in hermaphrodites.

We interpret the abnormalities seen in B to be the result of a transformation in its identity to that of Y. However, it is possible that B may simply be defective and degenerating gradually.

\section{Other phenotypes of mab-9}

Defects in $U$ and $F$ lineages The lineages of the minor male-specific blast cells $\mathrm{U}$ and $\mathrm{F}$ are abnormal in mab-9. The mutant $\mathrm{F}$ lineages may indicate a distinct function of mab-9 in specifying the identity of F. For U, however, there is evidence that the defects are purely a secondary result of the defective $B$ lineage.

Examples of the abnormal $F$ lineages seen in mab-9(e2410) males are shown in Figure 4. The divisions of the ventral daughters of F.l and F.r, which normally occur 2 hr after their parents divide, do not occur. Instead, either of F. $\left({ }_{\mathrm{r}}^{1}\right) \mathrm{v}$ or F. $\left({ }_{\mathrm{r}}^{1}\right) \mathrm{d}$ may divide about $2 \mathrm{hr}$ later, concurrent with the divisions of U. $\left({ }_{r}^{1}\right) \mid a$. Because the F. $\left({ }_{r}^{1}\right) v$ division is the only characteristic of the F lineage differentiating it from that of $U$ laside from some of the terminal fates), its omission suggests that $F$ is transformed toward $U$. The loss of the F. $\left({ }_{r}^{1}\right) v$ division round does not require the presence of a transformed $B$ lineage,

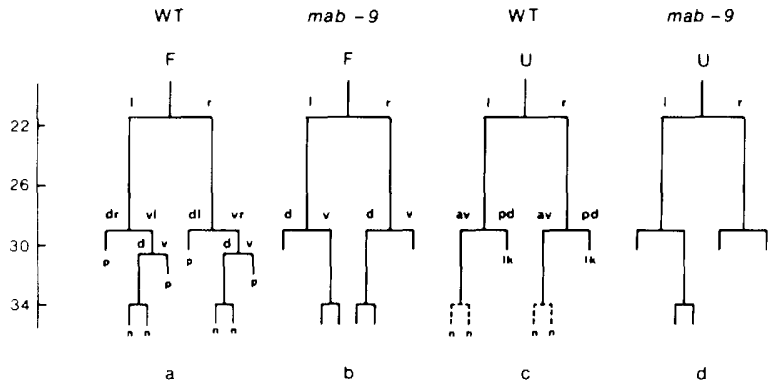

Figure 4. $\mathrm{F}$ and $\mathrm{U}$ lineages in mab-9 males. $|a|$ Wild-type $\mathrm{F}$ lineage. Terminal fates shown are: $(p)$ structural cell in proctodeum; $\{\mathrm{n}$ ) neuron. $(b)$ Mutant $\mathrm{F}$ lineage seen in mab-9. Based on observations of e1245 $(n=5) ;$ e1245/nDf3 (3); e2410 (5); $e 2410 / n D f 3$ (3). F. $\left.\left.\right|_{r} ^{1}\right) v$ was never seen to divide at the correct time $\left[1.5 \mathrm{hr}\right.$ after the divisions of $\left.F .\left({ }_{\mathrm{r}}^{1}\right)\right]$. Instead, either $\mathrm{F} .\left({ }_{\mathrm{r}}\right) \mathrm{v}$ or F. $\left({ }_{r}^{1}\right) d$ divided at the normal time of the F. $\left(\left.\right|_{r} ^{1}\right) v d$ and U. $\left({ }_{r}^{1}\right)$ a divisions $(34 \mathrm{hr})$. About half the F. $\left({ }_{\mathrm{r}}^{\mathrm{r}}\right)$ lineages aborted after the F. $\left({ }_{\mathrm{r}}^{1}\right)$ division. Occasionally, both daughters of $F .\left({ }_{\mathrm{r}}^{1}\right)$ divided (symmetrization). (c) Wild-type $U$ lineage. The divisions of $U .\left({ }_{r}^{1}\right)$ a are variable: usually one divides but occasionally both divide; it is not known whether in some wild-type animals neither divides. (lk) Killer of linker cell. (d) Mutant U lineage seen in mab-9. The only abnormality is that there is asymmetry reversal of the divisions of U. $\left({ }_{r}^{1}\right)$ : out of the five U. $\left({ }_{r}^{1}\right)$ lineages in which one daughter divided, two were reversed and three were nonreversed. In 5 of 10 worms, none of the 4 U. $\left({ }_{\mathrm{r}}^{1}\right)$ daughters divided. This may indicate a symmetrization of the U. $\left({ }_{r}^{1}\right)$ division, but the variability of the lineage in wild type makes this conclusion weak. mab-9 mutations cause a low penetrance effect on the cell $\mathrm{K}^{\prime} . \mathrm{K}^{\prime}$ is the nondividing sister cell of the blast cell $\mathrm{K}$, which divides once in late $\mathrm{L} 1$ in both sexes. In less than $5 \%$ of mab-9 males $\mathrm{K}^{\prime}$ divides once in late L1, i.e, similarly to its sister. This effect on $\mathrm{K}^{\prime}$ is mimicked at low penetrance by killing the $\mathrm{B}$ cell $/ \mathrm{K}^{\prime}$ divided in $1 / 19$ males in which $\mathrm{B}$ had been ablated in early L1). Moreover, out of $50 \mathrm{~L} 4$ or adult $m a b-9(e 1245)$ and $50 m a b-9(e 2410)$ hermaphrodites examined, none had a divided $\mathrm{K}^{\prime}$. It seems likely that the rare division of $\mathrm{K}^{\prime}$ is a nonspecific secondary effect and does not represent a separate function for $m a b-9$.

because it was still lost when B was killed in two mab-9(e2410) males. In contrast, the polarity reversals may be secondary effects, since they are mimicked by ablating $B$ (see below).

The $U$ lineage in mab-9 males shows one abnormality: the polarity of the U. $\left({ }_{r}\right)$ division is sometimes reversed, so that $U .\left(\left.\right|_{r} ^{1}\right) p$ is seen to divide instead of $U .\left({ }_{r}^{1}\right) a$. This reversal also is seen in worms in which the $B$ cell has been ablated (see below), and therefore is probably a secondary effect of the abnormal cellular environment caused by the mutant B lineage. The linker cell is sometimes not killed in mab-9, implying that the terminal fates of $U$ may be affected [U. $\left.\right|_{r} ^{1}$ ) p are the killers of the linker cell], although this could be simply a result of mispositioning of the cells.

Morphogenetic defects in the male tail fan The morphogenetic processes that produce the fan of the male tail occur late in L4, just prior to the final molt to adult. In some mab-9 males, the initial steps in morphogenesis (ventral cytoplasmic retraction, ray elongation) occur correctly, but subsequent stages are blocked by a bulging 
out of cytoplasm at the posterior end of the tail /see Fig. 5). The resulting adult fan is club-shaped and irregular. The penetrance of this defect is given in Table 1. There is some evidence from ablation experiments (see below) that this phenotype may be a secondary effect of the abnormal internal tail lineages.

Uncoordinated phenotype in both sexes mab-9 worms of both sexes are slightly but distinctly uncoordinated (Unc), from Ll onward. This is strongest in $m a b-9(e 2410)$ and weakest in $e 2376$, and is seen in all heteroallelic combinations. $e 2410$ worms kink when moving backward, but they kink less when moving forward; this is not worse in $e 2410 / n D f 3$ worms. The cause of the Unc phenotype is unknown: the numbers and placement of embryonic and postembryonic neurons in the ventral cord and tail ganglia are normal.

\section{Effects of laser ablation of $B$ in wild type}

Are the lineage defects seen in $F$ and $U$ and the tail fan morphogenesis defects secondary results of the abnormal B lineage in mab-9, or do they reflect separate functions of mab-9 in addition to specification of $\mathrm{B}$ cell fate? To approach this question, we attempted to pheno- copy these phenotypes by killing the B cell by laser ablation in wild-type worms (see Discussion for caveats). Sulston and White (1980) suggested, on the basis of a small number of ablations, that killing any one of the internal blast cells had no effect on the others. It was important to try and confirm this with more animals and a different laser setup (see Materials and methods).

Effects on $F, U$, and $K^{\prime}$ lineages The B cell was killed in 12 early $\mathrm{Ll}$ wild-type males as described in Materials and methods, and the F lineage followed. In 9 of the 12, the $U$ lineage was also followed. Abnormalities were seen in the later parts of both lineages (see Fig. 6 for details). The effects of killing $B$ on the $F$ lineage may be described as the result of two variable operations on the wild-type lineage pattern. First, the unequal and asymmetric divisions in the second and third rounds of the $F$ linage (divisions of F. $\left(\begin{array}{l}l \\ r\end{array}\right)$ and $F .\left({ }_{r}^{l}\right) \mathrm{v}$, respectivelys] are reversed, so that the polarity becomes almost random $(40 \%$ reversed and $60 \%$ normal $)$. Second, the lineage tends to abort after the second round. Only 2 of the 24 F. $\left(l_{r}\right)$ lineages examined were wild type. Therefore, B descendants appear to provide important (but not essential) cues for the polarities and proliferation in F. Similar reversals of the asymmetric divisions of $U .\left(\frac{1}{r}\right)$ were also
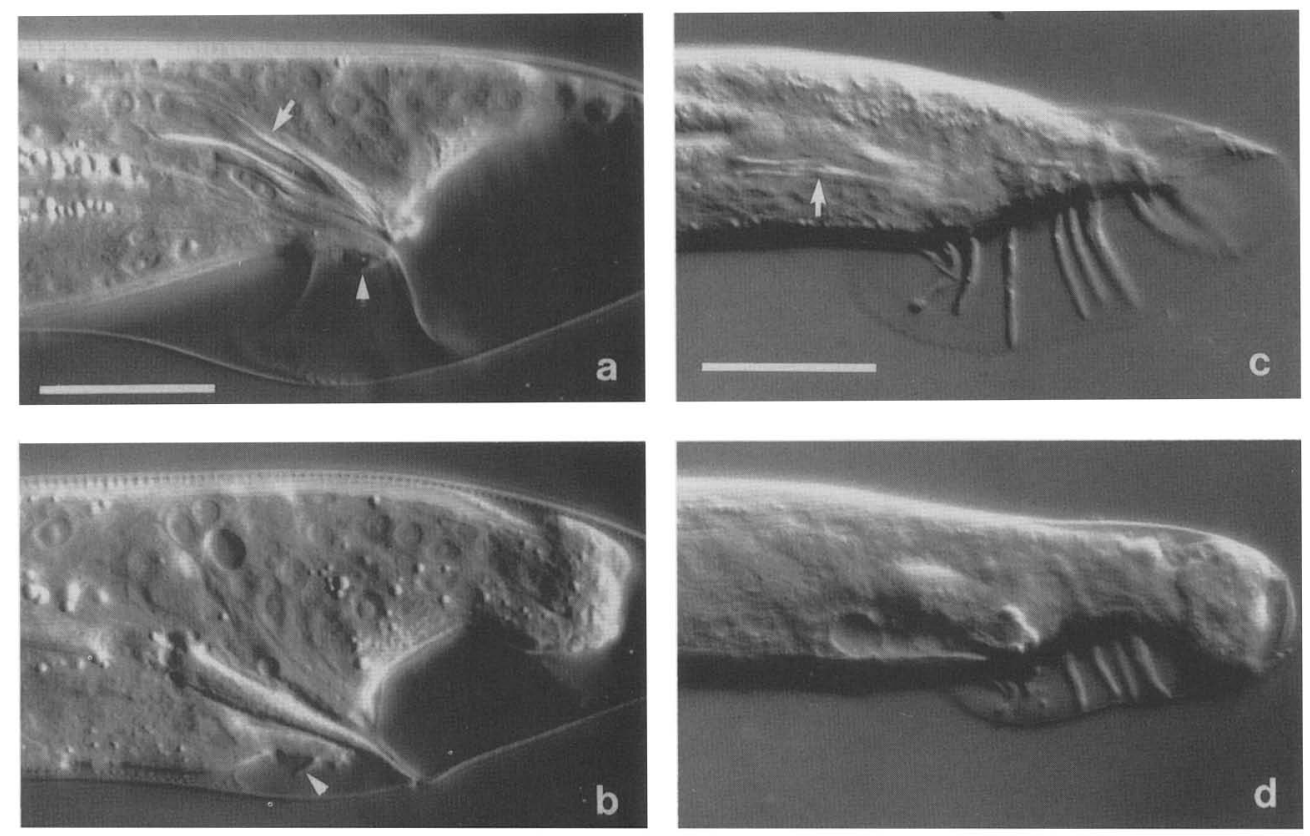

Figure 5. Nomarski photomicrographs of male tail morphogenesis in wild type and mab-9. $|a|$ Lateral view, focal plane slightly left of midline. Wild-type male tail in late $\mathrm{L} 4(\sim 45 \mathrm{hr})$. The ventral retraction of the fan has begun; the rays are extended (out of focus). Note spicules (arrow) and hook (arrowhead). Scale bar, $10 \mu \mathrm{m}$. (b) Similar view to that in $a$, mab-9(e2410) slightly younger male. Spicule traces are completely absent and the interior of the tail is disorganized. Anterior fan morphogenesis is relatively good and a reasonable hook is made (arrowhead), but cytoplasm has started to bulge out from the posterior end. Scale as for $a$. (c) Lateral view, young adult wild-type male. Spicules (arrow) are slightly out of the plane of focus. Scale bar, $25 \mu \mathrm{m}$. (d) View as for c, mab-9(e2410) male. Scale bar, $25 \mu \mathrm{m}$. Note absence of spicules and posterior fan bulge. Males without the posterior bulge show minor fan morphogenetic defects such as small fans, blobby or fused rays; in about $5 \%$ of males the fan is virtually wild type. In many males the rectum or gut everts from the cloaca, killing the worm in the $\mathrm{L} 4$ molt or soon after; in some there is a sclerotized blob resulting from partial eversion. In some animals small patches of sclerotization may be found, possibly representing an incomplete loss of B terminal fates or simply a result of the abnormal cellular environment in mab-9 tails. Occasionally one or two elongate sclerotized structures recognizable as spicules are made (see Table 1 for penetrance). 


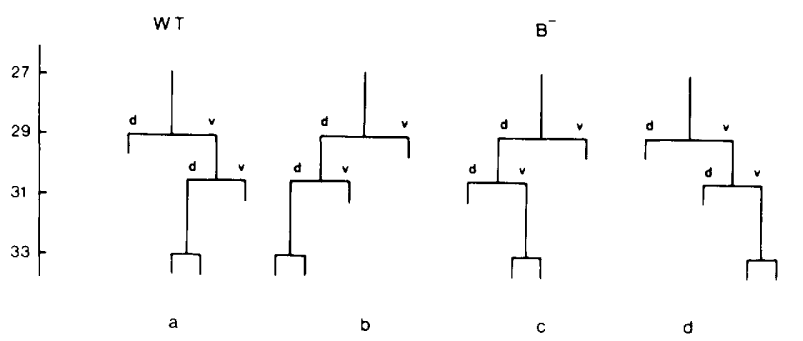

Figure 6. Effects of killing B on the F lineage. $(a)$ Wild-type F. $\left({ }_{r}\right)$ lineage for comparison. $(b-d)$ Examples of $F \cdot\left({ }_{r}\right)$ lineages seen in males where $B$ was killed in Ll. The F lineage was always normal up to the division of $F .\left({ }_{r}^{1}\right)$, so only the later lineages are shown. Times of rounds of division, when they occurred, were the same as in wild type. A total of $24 \mathrm{~F} .\left({ }_{\mathrm{r}}^{1}\right)$ lineages were followed. The simple omission of the division round $2 \mathrm{hr}$ after the F. $\left({ }_{\mathrm{r}} \mathrm{r}\right)$ divisions, as seen in mab-9, was not observed. Only 2 of $24 \mathrm{~F} .\left({ }_{\mathrm{r}}\right)$ lineages were wild type $(a)$. In 11 of $24, \mathrm{~F} .\left(\left.\right|_{\mathrm{r}} ^{1}\right)$ was reversed $\langle b|$; in 4 of $\left.11 \mathrm{~F} .{ }_{\mathrm{r}}{ }_{\mathrm{r}}\right) \mathrm{d}$ was also reversed $(c)$; in 3 of the 11 , the lineage stopped after the division of $F .\left({ }_{r}^{1}\right) d$, and in 1 F. $\left({ }_{r}^{1}\right)$ d divided symmetrically, with both of its daughters dividing. In five lineages, the lineage stopped at the division of F. $\left({ }_{r}\right)$ v. In two lineages, the division of $F .\left({ }_{r}^{1}\right) v$ only was reversed (d). In two, both daughters of F. $\left({ }_{r}^{1}\right)$ divided (symmetrisation). In one, the lineage aborted after the division of $F .\left({ }_{r}^{1}\right)$. In the same 12 worms, 18 U. $\left({ }_{\mathrm{r}}^{1}\right)$ lineages were followed: in 4 of 18 , neither daughter divided; in 7 of $18 \mathrm{U} .\left({ }_{\mathrm{r}}^{1}\right)$ a divided; and in 7 of $18 \mathrm{U} .\left({ }_{\mathrm{r}}^{1}\right) \mathrm{p}$ divided (asymmetry reversal). The frequency at which $F .\left(\left.\right|_{r} ^{1}\right)$ and $\mathrm{U} .\left({ }_{\mathrm{r}}\right)$ division asymmetry is reversed is roughly similar (about $40 \%)$.

seen, at about the same frequency (i.e, nearly random polarity) as in mab-9. In one of the males, $\mathrm{K}^{\prime}$ divided dorsoventrally. The $\mathrm{Y}$ lineage was wild type in all worms lineaged.

Effects on fan morphogenesis From a total of 19 males in which B was killed in early L1 (including the 12 lineaged), fan morphology was severely abnormal in only one male. In this worm, the defective fan resembled that seen in mab-9, that is, a distinctive bulge of cytoplasm at the posterior end of the tail. The fans of the other 18 worms were essentially wild type, although some were a little smaller than normal. In most worms, the gut partially everted at the cloaca during L4, producing a refractile blob as in mab-9 males. Killing both $\mathrm{B}$ and $\mathrm{F}$ produced a mab-9-like fan defect in three out of six worms. The morphogenetic defects of mab-9 male tails (which are only $60 \%$ penetrant in the strongest allele, e2410) may therefore be accounted for by secondary effects of incomplete internal development.

\section{Absence of interaction of mab-9 with lin-12}

One assay for the transformation of $B$ into $Y$ would be to analyze the phenotype of double mutants of mab-9 with mutations in a second gene required for the $Y$ lineage. If $B$ were transformed into $Y$, then the second mutation would be expected to affect the 'ectopic' Y cell as well as the normal one.

Null mutations in lin-12 ['lin-12(o)'] transform $\mathrm{Y}$ into a neuron in males (Greenwald et al. 1983). If B is trans- formed fully into $\mathrm{Y}$ in $m a b-9$, then into the mab-9; lin-12(o) double mutant the ectopic Y might be further transformed into a neuron. Some reduction in proliferation might still be expected in the case of incomplete transformation.

To test this prediction, double mutant strains were made with mab-9(e1245) and the lin-12 putative null $n 137 n 720$. Of six e1245;lin-12(o) males lineaged, none showed any alteration in $B$ lineage from the range normally seen in mab-9 males, although very late effects cannot be excluded. The transformation of $\mathrm{Y}$ to neuron occurred as in In-12(o) alone. Spot checks on several L4 males of the above genotype showed that the approximate number of nuclei in the $B$ region was similar to that seen in mab-9 alone. Therefore, the prediction is not fulfilled; the implications for the functions of mab-9 and $l i n-12$ are discussed below.

\section{Discussion}

The loss-of-function phenotype of mab-9

Several lines of evidence suggest that the strong mutant phenotypes described above are those resulting from loss of mab-9 gene function. First, the mutant phenotypes of the alleles e1245 and e2410 do not appear enhanced when placed over a deficiency that spans mab-9. The phenotypes are variable in both alleles and deficiency heterozygotes, but phenotypic variability is not in itself evidence that a mutation is non-null (see Ferguson et al. 1987; Shen and Hodgkin 1988). Second, in a noncomplementation screen in which lethal or sterile nulls could have been recovered, none was found (although the screen was too small to make this a strong argument). Third, the strong allele $e 1245$ is a nonsense mutation: such mutations are usually strong hypomorphs or nulls, although exceptions exist (e.g., the weak tra-1 allele e1825; Hodgkin 1987); e1245 is unlikely to be null as it is slightly weaker than $e 2410$. Finally, the frequency of isolation of mab-9 alleles $\left(3 \times 10^{-4}\right)$ is within the normal frequency range for isolation of simple reduction-of-function alleles in C. elegans.

\section{Primary and secondary effects of mab-9 mutations}

We interpret the lineage abnormality in mab-9 males as being the result of a transformation in the identity of the $B$ cell to the $Y$ cell. By several lineage criteria, the abnormal B lineage in mab-9 males has the characteristics of a Y lineage, although rarely a perfect one. The abnormal morphology of the B cell in mab-9 hermaphrodites also can be interpreted as being the result of a B-to$Y$ transformation. The defective specification of the $B$ cell in both sexes can account for most of the gross phenotypes seen in mab-9 worms.

Several other mutant phenotypes have been noted in mab-9 worms. As described above, the effects on $U$ and $\mathrm{K}^{\prime}$ are probably purely secondary effects of mab-9 because they can be mimicked by laser ablation of the $B$ cell. The tail fan morphogenetic defects also are copied at low frequency by killing $B$ (and at higher penetrance if 
both $\mathrm{B}$ and $\mathrm{F}$ are killed). Therefore, it is feasible for abnormalities in the internal lineages to result in aberrant fan morphogenesis, although at lower penetrance than in the mutant.

The abnormal $F$ lineage in mab-9 males resembles that of $U$, in that it omits the third round of divisions. The case for this being the result of an F-to-U transformation is weaker than that for $B$ and $Y$, because the wild-type $F$ and $U$ lineages are very similar. As shown above, killing $B$ can produce a variety of abnormal $F$ lineages, but the type consistently seen in mab-9 has never been seen. The loss of the third round still occurs if $B$ is killed in a mab-9 background.

Attempts to produce a phenocopy by laser ablation, and thus decide whether the phenotype in question is a primary or secondary consequence of mutation, can only give an answer if a phenocopy is produced. A failure to phenocopy could be either because the effect was not secondary, or because laser ablation has not mimicked the primary defect precisely. The latter is clearly possible because the debris resulting from the ablation of $B$ is different from the ectopic Y cells found in mab-9. The simplest interpretation of the ablation data (ignoring issues of penetrancel is that the effects of mab-9 mutations on $\mathrm{U}, \mathrm{K}^{\prime}$, and fan morphology are all secondary to the $\mathrm{B}$ defect. The $\mathrm{F}$ lineage defects in mab-9 may result from secondary effects of $B$ laffecting asymmetric divisions and proliferation) superimposed upon a transformation of $\mathrm{F}$ to $\mathrm{U}$. A less ambiguous answer might be provided by mosaic analysis (Herman 1987).

It is interesting that the descendants of B appear to be involved in setting up the correct polarity of asymmetrical divisions in the $F$ and $U$ lineages. The reversals seen are unlikely to be the result of accidental damage to $\mathrm{F}$ or $\mathrm{U}$ because the divisions are not delayed from wild type. B progeny form most of the cellular environment in the L3 male tail, so such minor effects are unsurprising. Polarity reversal in response to ablation has also been seen in the male lateral hypodermis /Sulston and White 1980) and in the somatic gonads of both sexes (Kimble 1981). The abortive $F$ and $U$ lineages also seen may reflect controls on proliferation or simply damage to $\mathrm{U}$ and $\mathrm{F}$.

\section{The function of lin-12 in $Y$ and $B$}

In lin-12(o) males, $\mathrm{Y}$ is transformed into a neuron (Greenwald et al. 1983). From the reciprocal phenotype in lin-12 dominant gain-of-function alleles, where the DA9 neuron is transformed into an extra $\mathrm{Y}$ blast cell, the fate of the extra neuron made in the null mutant is inferred to be DA9 (the Y and DA9 cells are lineage homologs; Sulston et al. 1983). The requirement of lin-12 for $\mathrm{Y}$ suggested the use of lin-12 mutations to assay the degree of $\mathrm{Y}$ identity of the transformed $\mathrm{B}$ cell; if $\mathrm{B}$ is transformed to $\mathrm{Y}$ in mab-9 and $\mathrm{Y}$ transformed to DA9 in lin-12, one might predict that in the double mutant $\mathrm{B}$ would be transformed into DA9. However, in the mab-9;lin-12(o) double mutant, B continues to execute a $\mathrm{Y}$-like lineage.
There are two possible explanations of this result. One is that $\mathrm{B}$ is not transformed fully into $\mathrm{Y}$ in $m a b-9$, and hence is not susceptible to further transformation in lin-12(o). This is conceivable, given the variable nature of the transformation. The alternative is that lin-12 is not required for the terminal fate of $Y$ (i.e., the particular characteristics of its lineage), but is required for a prior choice of developmental pathway.

We find the second explanation more attractive. 1 in -12 is required for the correct choice of cell fate for many different types of cell: Its common function is to choose between two alternative developmental pathways (Greenwald et al. 1983). This function is prior to the specification of the terminal fates of the cells that followed these paths. lin-12 function is therefore not required for the $\mathrm{Y}$ fate of a transformed B cell in mab-9, as $B$ did not make a lin-12-dependent choice earlier on. The terminal fate of $Y$ requires genetic information extant in the double mutant, and hence the ectopic $\mathrm{Y}$ is unaffected. However, this is a negative result, so any interpretation must be weak.

\section{The wild-type function of mab-9}

A tentative conclusion of the foregoing analysis is that the wild-type function of the mab-9 gene is to specify the identity of $\mathrm{B}$ as distinct from Y. mab-9 may also be responsible for a similar identity choice for $F$ versus $U$, although the evidence is unavoidably weaker. A hypothetical full F-to-U transformation would consist of the omission of only one division round. This is what is seen in mab-9 mutants.

These functions appear to be required in both sexes. Despite the far more striking male phenotype, mab-9 does not play any direct role in the generation of sexual dimorphism: the phenotypes are not the result of sexual transformation. mab-9 need not be under any sex-specific regulation; an independent function (tra-1 or some 'downstream' genel must control the choice of male (blast cell) versus hermaphrodite (structural) fates.

\section{Comparison with other cell lineage genes}

The most striking aspect of the lineage phenotype in mab-9 males is its specificity, being confined to two neighboring blast cells in the dorsal roof of the rectum. To date mab-9 is the only gene whose lineage requirements are confined to the male-specific blast cells. A separate function for mab-9 in the locomotory system (possibly in the nervous system) is implied by the uncoordinated phenotype of mutants.

Hitherto, most cell lineage genes in C. elegans have been found to be highly pleiotropic (Horvitz 1988). Such genes may be required in a set of repeated sublineages (e.g., mab-3; Shen and Hodgkin 1988), for regional differentiation within a set of similar precursors (e.g., mab-5; Kenyon 1986), or have general roles in several lineages (e.g., lin-14; Ambros and Horvitz 1984). It is possible that many more genes with local roles in cell determination exist (e.g., ham-1; Desai et al. 1988), but that are 
unidentified as yet because of their subtle phenotypes: mab-9 would not have been picked up on the basis of its hermaphrodite phenotype.

The cause of the Unc phenotype has not been pursued here. Several precedents exist in C. elegans for genes having roles in both cell lineage and neuron terminal fate, such as unc-86 (Desai et al. 1988). This is reminiscent of the dual function of several Drosophila genes in segmentation and neuron identity (e.g., fushi tarazu; Doe et al. 1988|.

Several genes are known which are required to differentiate otherwise identical sisters or homologs (in pairs or series). A good example is lin-12 (Greenwald et al. 1983|, which is required for the correct choice of alternative cell fates in nine pairs of homologs, one pair of sisters (B.pa/B.pp), and two series of analogs (vulval and preanal equivalence groups). For some of these functions, there is a simple rationale: The cells involved are initially equivalent and acquire their fates by interaction with one another.

In the embryonic lineage, $\mathrm{U}$ and $\mathrm{F}$ are sister cells but $\mathrm{B}$ and $\mathrm{Y}$ bear no simple genealogical relation; $\mathrm{B}$ and $\mathrm{U}$ are lineage homologs (Sulston et al. 1983). It is significant that the relative positions of the $F, U$, and $B, Y$ blast cell pairs are analogous (see Fig. 1); the $U$ and $F$ lineages are related and the geometry of the division axes in the early lineages of $\mathrm{B}$ and $\mathrm{Y}$ is clearly related (Fig. 2a and $\mathrm{b}$ ). Therefore, mab-9 acts on the posterior member of one sister pair and one pair of nonhomologous analogs; the common characteristic of these pairs is their spatial disposition, straddling the rectum.

If $m a b-9$ is required to differentiate the posterior members of the two cell pairs in the rectum, why should such a function be necessary? The sisters $F$ and $U$ are possibly initially equivalent, but why should $\mathrm{B}$ and $\mathrm{Y}$ be similar? Despite their disparate genealogies, $\mathrm{B}$ and $\mathrm{Y}$ are born next to one another in embryogenesis, forming a pair on the left of the prospective anus, roughly symmetrical to that of $\mathrm{F}$ and $\mathrm{U}$ on the right. There is no evidence for any interaction between them in choice of fate. Some extrinsic mechanism may exist determining that $B$ and $\mathrm{Y}$ are similar, and it is possible that their origin is simply another 'developmental fossil' buried in the embryonic lineage.

\section{Materials and methods}

\section{Strains and alleles}

Nematode strains were cultured by standard techniques (Sulston and Hodgkin 1988). Mutations used are described in Hodgkin et al. (1988) or below. Alleles used were as follows. LGII: egl-26(e1952) (pka egl-48), mab-9(e1245am, e2376, e2410), lin-42(n1089) (Z. Liu and V. Ambros, pers. comm.), sup-9(n180), lin-31(n301), unc-85(e1414am), bli-2(e768); LGIII: unc-93(e1500), unc-36(e251), sup-5(e1464), dpy-19(e1259ts), unc-32(e189), lin-12(n137n720, n676n909am), tra-1(e1099); LGIV: him-8(e1489), dpy-20(e2017am); LGV: him-5(e1490), dpy-11(e224); LGX: sup-7(st5), sup-21(e1957). Rearrangements used were: LGII: $n D f 2, n D f 3$, and $n D f 4$ (Greenwald and Horvitz 1980).

\section{Strain construction}

Strain construction was performed generally by standard methods. To study the male phenotype of mab-9, it was convenient to use strains containing him -5 or him- 8 mutations, which increase the frequency of spontaneous male progeny to 33 and $37 \%$, respectively (Hodgkin et al. 1979).

In the construction of mab-9; 1 in-12 double mutants, the original strains constructed contained the marker mutation unc-32(e189) tightly linked to lin-12. Males of this strain were very ill and usually died in early larval development, precluding lineage analysis. The larval lethality was reduced by introducing an additional wild-type copy of the unc-32 gene into the strain. This copy derives from the transgenic strain CB4442 of genotype unc-32(e189); eIs16[unc-32(+)], generated by J. Sulston (pers. comm.). CB4442 was constructed by transformation rescue of unc-32(e189) by microinjection of the cosmid ZK637, using a modification of the procedure of Fire (1986). The transforming DNA has integrated on LGV, close to $d p y-11$ (data not shownI. Males of genotype unc-32; $d p y-11 /$ els16 were mated with mab-9; unc-36; dpy-11 hermaphrodites and the wild-type cross-progeny males were mated with mab-9; unc-32 lin-12/ unc-36; him- 8 hermaphrodites. The required genotype was generated by picking the appropriate phenotypes in subsequent generations. The full genotype of the strain analyzed was mab-9(e1245);unc-32 lin-12(n137n720)/unc-36; him-8; els16; an analogous strain with the $\operatorname{lin}-12$ allele $n 676 n 909$ am was also spot-checked.

Deficiency heterozygotes were made by mating mab-9/+ males with $n D f 3 /$ lin-31 bli-2 hermaphrodites.

\section{Isolation of mab-9 alleles and mapping}

All three alleles of mab-9 were induced by ethyl methane sulfate (EMS) mutagenesis. The first allele, e1245am, was isolated in a screen for mating-defective males (Hodgkin 1983; $n=$ number of $\mathrm{Fl}$ chromosomes screened $=2238$ | e2376 was found in a clonal screen for mab mutations linked to sup.9 $\langle n=1456)$. Mutagenized sup-9; unc-93; him-5 males were mated to unc-93; dpy-11, him-5 hermaphrodites and the Unc non-Dpy cross progeny cloned. From each F2 brood one nonUnc (i.e., Sup) hermaphrodite was cloned and its male progeny scored. e2410 derives from a clonal noncomplementation screen $(n=4465)$ using sup. 9 as a flanking marker. Mutagenized sup-9; unc-93; him-5 males were mated to mab-9(e1245); unc-93; dpy-11, him-5 hermaphrodites and the Unc non-Dpy cross-progeny cloned. F2 broods were screened for Mab Unc males; sibling non-Unc (Sup) hermaphrodites were picked from any putative isolates.

mab-9 had been mapped previously to the left arm of LGII (Hodgkin 1983). By three-factor and deficiency mapping, mab-9 was further localized to the egl-26-lin-42 interval (Fig. 7).

\section{Amber suppression}

The phenotype of mab-9(e1245) is suppressed by one or two copies of the amber suppressor mutation sup-5(e1464) \{Wills et al. 1983). A strain of genotype mab-9; sup-5; him-5 was constructed by mating sup-5/dpy-19 males with mab9(e1245); dpy-19; him-5 hermaphrodites. Non-Dpy F1 hermaphrodites were picked; from F2 broods segregating Mab males, non-Dpy sibling hermaphrodites were picked. Some of these worms segregated Dpy Mab and non-Dpy non-Mab males, that is, the parent was of putative genotype mab-9(e1245); sup5/dpy-19; him-5. Strains were isolated from these animals which were slow-growing (the phenotype of homozygous sup-5) 


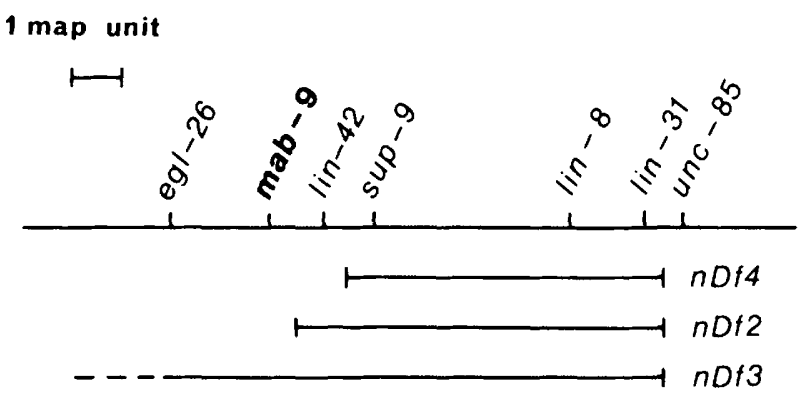

Figure 7. Map of the mab-9 region on the left arm of LGII. The order with respect to sup -9 was found by three-factor crosses: Among the progeny of heterozygotes of genotype mab-9(e1245)/sup-9 lin-31; unc-93; him-5, 10 of 10 Lin nonSup recombinants and 0 of 34 Sup nonLin recombinants picked up $m a b-9$, putting mab-9 to the left or very close to the right of sup-9. Two-factor crosses gave a distance from mab-9 to sup-9 of $2.2 \mu$ [from the ratios of male phenotypes in the progeny of mab-9 sup $-9 /++$; unc-93; him-5 : 343 nonMab nonSup (nonrecombinant), $3 \mathrm{Mab}$ nonSup and 7 Sup nonMab recombinants]. $m a b-9$ is ordered with respect to $l i n-42$ by three-factor data: $18 / 18$ Sup nonLin recombinant progeny from mab-9(e1245)/lin-42 sup-9; unc-93; him-5 parents picked up mab-9. Order with respect to egl-26 was given by three-factor data: 1 of 7 Sup nonEgl recombinants from e1245/egl-26 sup9; unc-93; him-5 parents failed to segregate Mab-9 males. The order of sup-9, lin-42, and mab-9 is confirmed by the deficiency mapping.

and did not throw Dpy or Mab worms, i.e., putative mab-9(e1245); sup-5; him-5 homozygotes. Homozygosity for mab-9 was confirmed by complementation. The other strong allele, e2410, was tested by a similar scheme and was not suppressed.

The lineage defect in e1245am males is suppressed strongly by sup-5. Good spicules and gubernaculum were made in $66 / 67$ mab-9; sup-5; him -5 males (assayed at $20^{\circ} \mathrm{Cl}$. The B lineage in one such male was followed to late $\mathrm{L} 3$ and found to be wild type. The tail fan morphogenetic phenotype is suppressed poorly: 35 of 67 males had an abnormal (mab-9) tail fan (this is not caused by sup -5 , which causes only a slight reduction in tail fan sizel. This may argue against the morphogenetic defects being secondary to the internal defects, but simply may reflect incomplete suppression. No suppressed males have been persuaded to mate. The Unc phenotype is variably suppressed.

Suppression of mab-9(e1245am) was tested also with the amber suppressors sup-7 (Wills et al. 1983) and sup-21 (Hodgkin 1985). To assay suppression of a male phenotype with two copies of the suppressors (which are X-linked) it was necessary to construct mab-9(e1245); tra-1/+; sup strains, in which sup. pression of the male phenotype of mab-9 was scored in Tra Sup XX animals. The mutations unc-85(e1414am) and dpy-20(e2017am) were present also as a linked marker for mab-9 and as an independent assay of suppression, respectively. For both suppressors, a strain of genotype mab-9(e1245) unc-85; tra-1/ + dpy-20; sup/+ was constructed. The heterozygous strains segregate non-Dpy males that can be scored for Mab. Suppression of $e 1245$ and $e 1414$ by sup-21 was strong, although not to wild type: good spicules were made in sup/sup and most sup/ + males (semidominant suppression); the Unc and morpho-Mab defects of e1245 were suppressed partially. Suppression of $e 1245$ by sup -7 was strong, but $e 1414$ was only weakly suppressed; thus, spicules were made, but they were crumpled as a result of the late lineage defects in 21414 males. The different suppression efficiencies may reflect tissue specificity of the suppressor tRNAs, as discussed by Kondo et al. \{1988).

\section{Cell lineage analysis}

Cell lineages were followed by Nomarski microscopy as described by Sulston and Horvitz (1977). In most animals the B, $\mathrm{U}, \mathrm{F}$, and $\mathrm{Y}$ lineages were followed from mid- $\mathrm{Ll}(8-10 \mathrm{hr})$ to the L3 molt ( $34 \mathrm{hr}$ ). Calibration of the timing of cell divisions was by reference to lineages not affected by mab-9 (usually the lateral and ventral hypodermis) and the times of molts. The characteristics of a cell lineage that have been used here and elsewhere (e.g., Sternberg and Horvitz 1986) as criteria for the transformation in fate of a blast cell are: times of cell divisions, sizes of cells, symmetry or asymmetry of divisions, total number of cells made (proliferation), and differentiated terminal fates (e.g., nuclear morphology under Nomarski microscopyl.

Apart from those mentioned in the text, all other lineages checked appeared to be wild type, apart from a low penetrance effect on $\mathrm{K}^{\prime}$ (see Fig. 4 legend). Lateral hypodermal (V5, V6, and $\mathrm{T})$ and ventral hypodermal (P10, P11, P12) lineages were followed until the L3 molt and appeared to be wild type; all 18 rays and the hook were made correctly. The mesodermal lineage $(M)$ was followed up to the penultimate divisions; the correct appearance of the diagonal sex muscles and dorsal coelomocytes demonstrates that mesodermal differentiation proceeds correctly.

\section{Laser ablations}

The laser setup was as described by Sulston and White (1980), except that the laser used was a Photochemical Research Associates LN1000/LN102 (nitrogen-laser-pumped). Ablations were carried out essentially as described by Avery and Horvitz (1987), on worms no more than $8 \mathrm{hr}$ after hatching (in which the $P$ cells had not migrated ventrally). Kills were verified the next day; if the nucleus of the ablated cell persisted, or if additional unwanted damage had been caused, the worm was discarded.

\section{Acknowledgments}

We would like to thank Michael Shen for many discussions. Leon Avery, Ellen Gottlieb, Michael Shen, and John Sulston provided critical comments on the manuscript. A.D.C. is the holder of an MRC research studentship.

\section{References}

Ambros, V. and H.R. Horvitz. 1984. Heterochronic mutants of the nematode Caenorhabditis elegans. Science 226: 409416.

Avery, L. and H.R. Horvitz. 1987. A cell that dies during wildtype C. elegans development can function as a neuron in a ced-3 mutant. Cell 51: 1071-1078.

Desai, C., G. Garriga, S.L. McIntire, and H.R. Horvitz. 1988. A genetic pathway for the development of the Caenorhabditis elegans HSN motor neurons. Nature 336: 638-645.

Doe, C.Q., Y. Hiromi, W. Gehring, and C.S. Goodman. 1988. Expression and function of the segmentation gene fushi tarazu during Drosophila neurogenesis. Science 239: 170175. 
Ferguson, E., P. Sternberg, and H.R. Horvitz. 1987. A genetic pathway for the specification of the vulval cell lineages of Caenorhabditis elegans. Nature 326: 259-267.

Fire, A. 1986. Integrative transformation of Caenorhabditis elegans. EMBO I. 5: 2673-2680.

Greenwald, I. and H.R. Horvitz. 1980. unc-93(e1500): A behavioural mutant of Caenorhabditis elegans that defines a gene with a wild-type null phenotype. Genetics 96: 147-164.

Greenwald, I., P. Sternberg, and H.R. Horvitz. 1983. The lin-12 locus specifies cell fates in Caenorhabditis elegans. Cell 34: 435-444.

Herman, R.K. 1987. Mosaic analysis of two genes that affect nervous system structure in Caenorhabditis elegans. Genetics 116: 377-388.

Hodgkin, J. 1983. Male phenotypes and mating efficiency in Caenorhabditis elegans. Genetics 103: 43-64.

- 1985. Novel nematode amber suppressors. Genetics 111: $287-310$.

1987. A genetic analysis of the sex-determining gene, tra-1, in the nematode Caenorhabditis elegans. Gen. Dev. 1: $731-745$

Hodgkin, J., H.R. Horvitz, and S. Brenner. 1979. Nondisjunction mutants of the nematode Caenorhabditis elegans. Genetics 91: $67-94$

Hodgkin, J., M. Edgeley, D. Riddle, and D.G. Albertson. 1988. Genetics appendix. In The nematode Caenorhabditis elegans (ed. W. Wood), pp. 491-584. Cold Spring Harbor Laboratory, Cold Spring Harbor, New York.

Horvitz, H.R. 1988. Genetics of cell lineage. In The nematode Caenorhabditis elegans (ed. W. Wood), pp. 157-190. Cold Spring Harbor Laboratory, Cold Spring Harbor, New York.

Kenyon, C.J. 1986. A gene involved in the development of the posterior body region of Caenorhabditis elegans. Cell 46: $477-487$.

Kimble, J.E. 1981. Alterations in cell lineage following laser ablation of cells in the somatic gonad of Caenorhabditis elegans. Dev. Biol. 87: 286-300.

Kondo, K., J. Hodgkin, and R.H. Waterston. 1988. Differential expression of five tRNA ${ }^{\mathrm{TP}} \mathrm{UAG}_{\mathrm{AG}}$ amber suppressors in Caenorhabditis elegans. Mol. Cell. Biol. 8: 3627-3635.

Shen, M.M. and J. Hodgkin. 1988. mab-3, a gene required for sex-specific yolk protein expression and a male-specific lineage in C. elegans. Cell 54: 1019-1031.

Sternberg, P.W. and H.R. Horvitz. 1986. Pattern formation during vulval development in C. elegans. Cell 44: 761-772.

Struhl, G. 1982. Genes controlling segmental specification in the Drosophila thorax. Proc. Natl. Acad. Sci. 79: 73807384.

Sulston, J.E. 1988. Cell lineage. In The nematode Caenorhabditis elegans (ed. W. Wood), pp. 123-155. Cold Spring Harbor Laboratory, Cold Spring Harbor, New York.

Sulston, J.E. and H.R. Horvitz. 1977. Postembryonic lineages of the nematode Caenorhabditis elegans. Dev. Biol. 56: 110156.

Sulston, J.E., D.G. Albertson, and J.N. Thomson. 1980. The Caenorhabditis elegans male: Postembryonic development of nongonadal structures. Dev. Biol. 78: 542-576.

Sulston, J.E. and J.G. White. 1980. Regulation and cell autonomy during postembryonic development of Caenorhabditis elegans. Dev. Biol. 78: 577-597.

Sulston, J.E., E. Schierenberg, J.G. White, and J.N. Thomson. 1983. The embryonic cell lineage of the nematode Caenorhabditis elegans. Dev. Biol. 100: 64-119.

Sulston, J.E. and J. Hodgkin. 1988. Methods. In The nematode Caenorhabditis elegans (ed. W. Wood), pp. 587-606. Cold Spring Harbor Laboratory, Cold Spring Harbor, New York.
Wills, N., R.F. Gesteland, J. Karn, L. Barnett, S. Bolten, and R.H. Waterston. 1983. The genes sup-7 X and sup-5 III of Caenorhabditis elegans suppress amber nonsense mutations via altered transfer RNA. Cell 33: 575-583. 


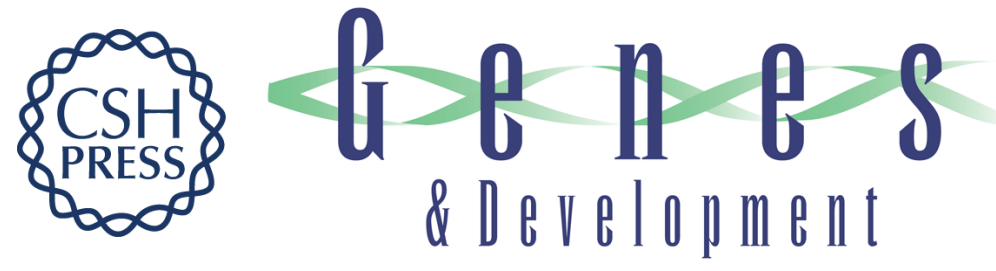

\section{The mab- 9 gene controls the fate of $B$, the major male-specific blast cell in the tail region of Caenorhabditis elegans.}

A D Chisholm and J Hodgkin

Genes Dev. 1989, 3:

Access the most recent version at doi:10.1101/gad.3.9.1413

References This article cites 24 articles, 10 of which can be accessed free at:

http://genesdev.cshlp.org/content/3/9/1413.full.html\#ref-list-1

License

Email Alerting

Service

Receive free email alerts when new articles cite this article - sign up in the box at the top right corner of the article or click here.

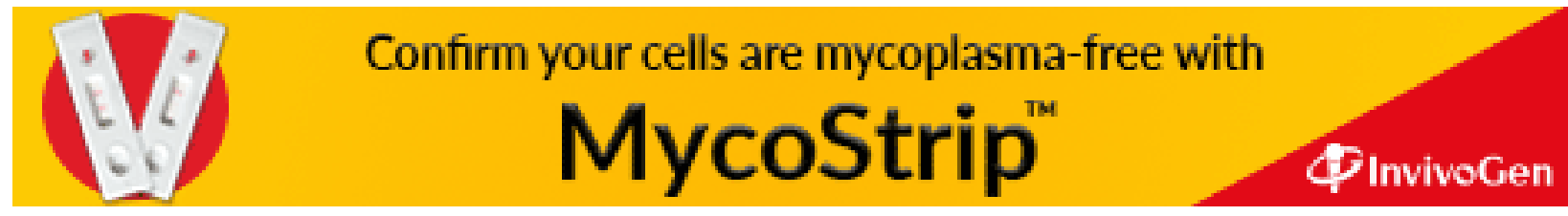

